\title{
Powder diffraction studies on proteins: An overview of data collection approaches
}

\section{Margiolaki ${ }^{1, *}$, J. P. Wright ${ }^{1}$, A. N. Fitch ${ }^{1}$, G. C. Fox ${ }^{1}$, A. Labrador', R. B. Von Dreele ${ }^{2}$, K. Miura ${ }^{3}$, F. Gozzo ${ }^{4}$, M. Schiltz $^{5}$, C. Besnard ${ }^{5}$, F. Camus ${ }^{5}$, P. Pattison ${ }^{1,5}$, D. Beckers $^{6}$, T. Degen ${ }^{6}$}

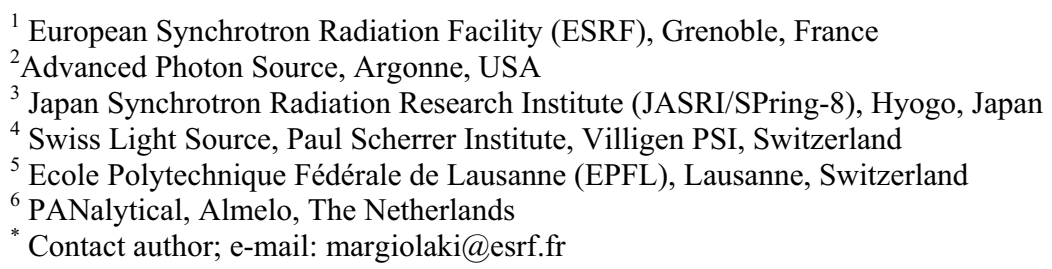

Keywords: powder diffraction, synchrotron radiation, protein crystallography

\begin{abstract}
Following the seminal work of Von Dreele, high quality powder X-ray diffraction studies on proteins are being established as a valuable complementary technique to singlecrystal measurements. Several studies using a variety of experiments approaches have been reported in the literature, including high-resolution studies employing parallel beam geometry and high intensity measurements using position sensitive detectors. The choice of the optimum instrumental configuration depends on a number of competing factors such as the amount of sample available, its radiation sensitivity, and the quality of the data required for data analysis, e.g. angular resolution, the extent of the data in d-spacing, or the number of patterns required to explore the protein's behaviour at different temperatures, or under different crystallisation conditions, etc. Here we discuss several advantages and disadvantages of different data collection methods followed for selected examples of small proteins.
\end{abstract}

\section{Introduction}

The investigation of protein crystal structures by high-resolution powder X-ray diffraction using synchrotron radiation is being established as a valuable complementary tool to traditional single-crystal techniques. There have already been several pioneering studies using powder diffraction methods to study proteins [1-5], and several more are in progress. The number of groups working in this area is growing and different instrumental configurations find applicability, including the instruments traditionally used for single crystal studies [3]. 
While most of the early studies were limited to dedicated powder diffraction instruments it is becoming increasingly clear that a wider range of instrumental configurations can give more useful information. A typical single crystal synchrotron beam line should also give a good quality powder pattern from a very small sample, if the X-ray beam is focused at the detector surface. Once a sample is found to be microcrystalline it is worthwhile to pursue an improved powder pattern. So far the majority of the successful powder examples have been well known proteins, used as test cases, but the time has come to attempt to expand the application of powder diffraction into new territories. One of the main barriers appears to be obtaining a useful powder pattern from a protein sample, as the unit cell volumes can be three orders of magnitude larger than those traditionally studied by powder methods. In this overview we describe a variety of instruments which are used at several major facilities and show useful information that can be obtained on a powdered protein sample in each case.

\section{Synergistic use of parallel beam geometry and area detector instruments}

One particularly important question that we want to address is the comparison of data collection schemes with high resolution analyser crystals on the one hand and multichannel position sensitive detectors (1 and 2-dimension) on the other. Whereas the former configuration offers maximum angular resolution, the latter provides substantially higher data collection rates, therefore, faster acquisition allowing for studies of particularly radiation sensitive systems. Data collection strategies and data quality from different instruments of four main synchrotron sources (ESRF, APS, SPring8 and SLS) as well as laboratory instrumentation (PANalytical) are discussed in the following sections.

\section{High angular resolution beam lines: Parallel beam geometry}

The high resolution powder diffraction beam line at ESRF based on an undulator source, ID31, has been extensively used in the last few years for structural studies of small proteins. This parallel beam geometry instrument is equipped with nine $\mathrm{Si}(111)$ analyser crystals. ID31 provides data with very high angular resolution, with a minimum instrumental contribution to the FWHM of around $0.003^{\circ} 2 \theta$ [6]. On the time scale of a few minutes, data suitable for extracting structural information and microstructural details can be obtained. Our experience is that this beam line is most effective for protein crystallography when operated with a wavelength above $1 \AA$. This strategy allows the very-low-angle (large-d-spacing) reflections to be measured accurately by moving them to higher diffraction angles, away from the beam stop, and to where the effect of low-angle asymmetry due to axial divergence is smaller. A bank of nine avalanche photodiode (APD) detectors ( $\mathrm{Si}$ based) is commonly used at this energy range. Because of the rapid data collection, high-speed spinning (3000rpm) of the samples is necessary in order to ensure adequate powder averaging. In order to deal with the particularly high sensitivity of proteins to radiation, ID31 is equipped with a fast capillary spinner that allows for sample translation along the axis of the diffractometer. Furthermore, high resolution instruments similar to ID31 are installed respectively at the X16C beam line at the National Synchrotron Light Source (NSLS) - Brookhaven National Laboratory, the Swiss-Norwegian beam line- BM01B (ESRF) and the materials science beam line at Swiss Light Source (SLS). X16C is installed on a bending magnet and equipped with a $\mathrm{Ge}(111)$ analyser crystal and was employed for the pioneering powder dif- 
fraction studies of sperm whale myoglobin [1a] and human insulin [1b]. BM01B is also set up on a bending magnet and is equipped with six $\mathrm{Si}(111)$ analyser crystals. The materials science beamline at SLS is based on a "minigap-wiggler" and has a three-coaxial axes diffractometer equipped with two detection systems that can be simultaneously operated: a high-resolution multicrystal analyser detector and a solid-state silicon microstrip detector [7]. The use of a bending magnet (BM01B/X16C) instead of an undulator (ID31) or a wiggler device (SLS) results in significantly reduced photon flux. Under comparable conditions, samples of protein PPE could be exposed at room temperature for several hours on BM01B before significant radiation damage occurred, whereas on ID31 their lifetime was limited to a few minutes. According to recent studies on macromolecular single crystals, radiation damage is distinctively correlated with the absorbed dose which can be expressed as a function of a number of parameters: the absorption coefficients of the atoms of the crystal, the number of molecules per asymmetric unit, the beam energy, flux, size and profile, the crystal size, and the total irradiation time [8]. Therefore, the lower photon flux can be advantageous. Moreover, in the case of PPE anisotropic lattice changes induced by radiation could be employed for distinguishing between neighbouring diffraction peaks which accidentally overlap resulting in better extracted intensities and detection of heavy atoms in protein derivatives [9].

Until now, proteins that have been successfully characterised using data collected at ID31 include, insulin, lysozyme from different sources, myoglobin, apoferritin, trypsin, elastase, thaumatin, thermolysin, glucose isomerase as well as small domains of muscle proteins (eg. $\mathrm{SH} 3$ domain of ponsin). The samples are usually slurries of polycrystalline proteins in saturated solvent packed in glass capillaries. The capillaries are centrifuged in order to achieve better packing of precipitate. Excess mother liquor is removed and the capillaries are sealed with wax to prevent protein dehydration. This approach for data collection requires at least 5$10 \mu \mathrm{l}$ of protein precipitant for each experiment. Figure 1 (upper) illustrates a Pawley fit [10], using the TOPAS software suite [11], to the ID31 data collected for a good quality sample of tetragonal hen egg white lysozyme (HEWL). In this case, the sample was loaded into a 1.5 $\mathrm{mm}$ glass capillary and centrifuged prior to data collection. Data were obtained at a wavelength of $1.250845(23) \AA$ and room temperature. In order to improve the counting statistics without compromising data quality, the capillary was translated to give a fresh region of sample as soon as radiation damage effects were evident in the peak positions and widths. Another interesting case corresponds to cubic insulin from bovine pancreas. Due to the high crystal symmetry the exact peak overlap of the powder diffraction data is quite severe and there are two molecules to be found in the asymmetric unit. Figure 1 (lower) shows a Pawley fit to the data collected at room temperature $(\lambda=1.250845(23) \AA)$. The fitted peak width for these data is about $0.01^{\circ}$ in $2 \theta$, including the low angle asymmetry due to axial divergence. In most of the cases of proteins studied up to now, indexing, intensity extraction via the Pawley approach, molecular replacement for determining the position and orientation of the molecule in the unit cell and structure refinements are performed using a single or multiple diffraction profiles depending on the data resolution (d-spacing) as well as the information 

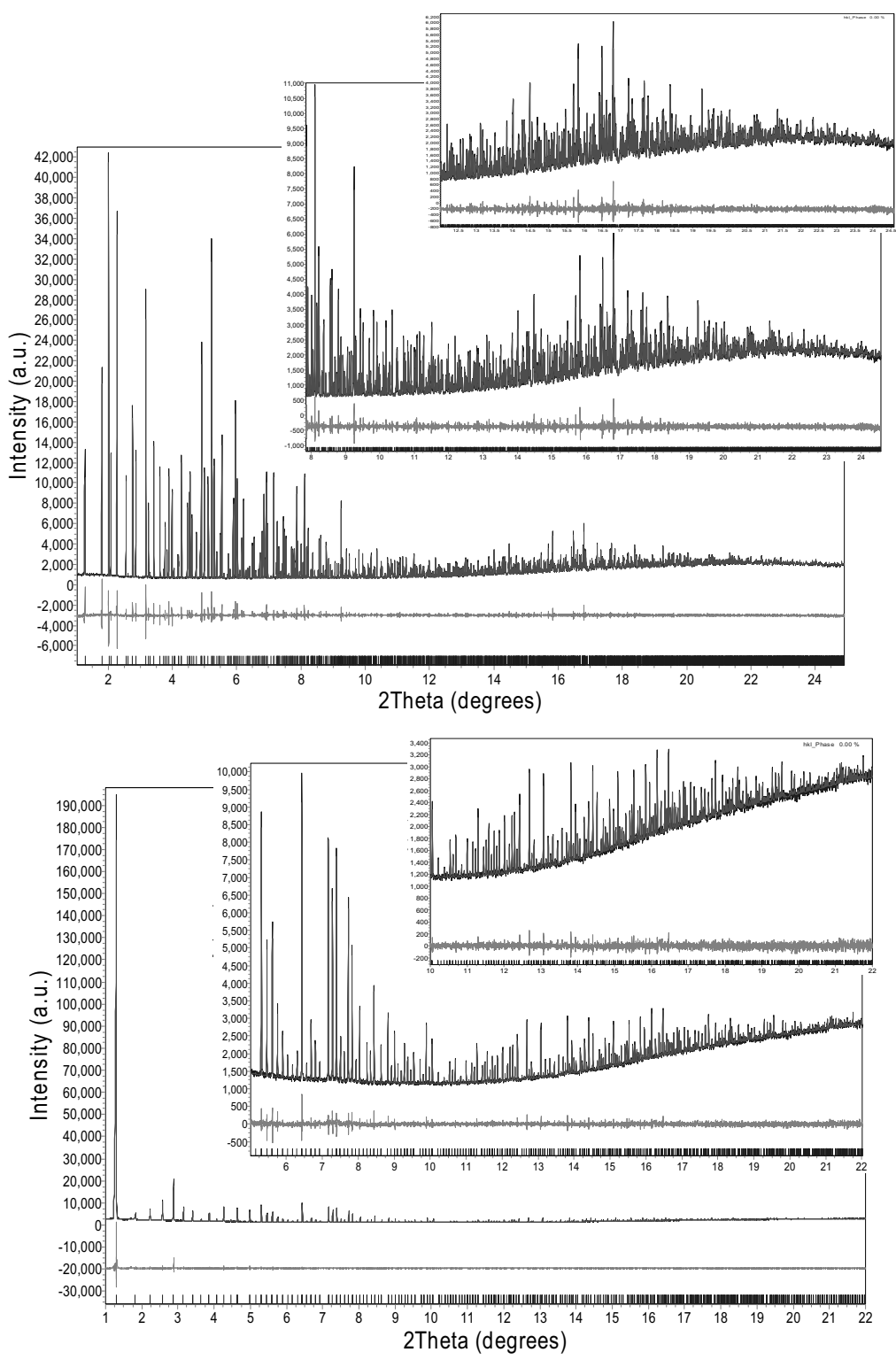

Figure 1: Pawley refinements of ID31 data $(\lambda=1.25 \AA, R T)$ of: (Upper) Tetragonal HEWL (sp. Group: $\left.P 4_{3} 222, a=79.2688(3) \AA, c=37.9573(2) \AA\right)$. In total, 2956 intensities were extracted up to $3 \AA$ resolution and (Lower) Cubic insulin from bovine pancreas (sp. group: $\left.I 2_{1} 3, a=79.0015(3) \AA\right)$. In total, 1957 intensities were extracted up to $3 \AA$ resolution. Insets: Magnifications of the high angle regions of the data. 
content of the powder profiles rulled by the crystal symmetry, sample crystallinity, the average size of the molecule and the lattice dimensions [2b].

\section{The microstrip detector (MYTHEN) at SLS}

The MYTHEN detector has been designed at the Paul Scherrer Institute detector group [7]. MYTHEN is a modular 1D detector, whose modules covers each approximately $5^{\circ}$ in $2 \theta$ and collects, in its present version, $60^{\circ}$ full diffraction patterns in times ranging between 1 and 10 seconds with the assembling of 12 modules. The MYTHEN intrinsic FWHM-resolution is $0.004^{\circ}$ and depends on the sample-to-detector distance $(760 \mathrm{~mm})$ and the silicon microstrip pitch $(50 \mu \mathrm{m})$. However, as in the case of an area detector described in the next section, the FWHM-resolution is influenced by the sample size although an improvement can be achieved at low $2 \theta$ angles by focusing the beam vertically on the detector instead of on the sample. The new generation microstrip detector, with reduced acquisition times by more than a factor of three, will allow for full diffraction patterns to be simultaneously acquired over $120^{\circ}$ in $2 \theta$. Preliminary measurements on HEWL have already been performed with particularly interesting results, no evidence of radiation damage was found: the high MYTHEN counting efficiency permitted the data to be acquired with a minimal radiation dose.

\section{High intensity beam lines: Area detectors}
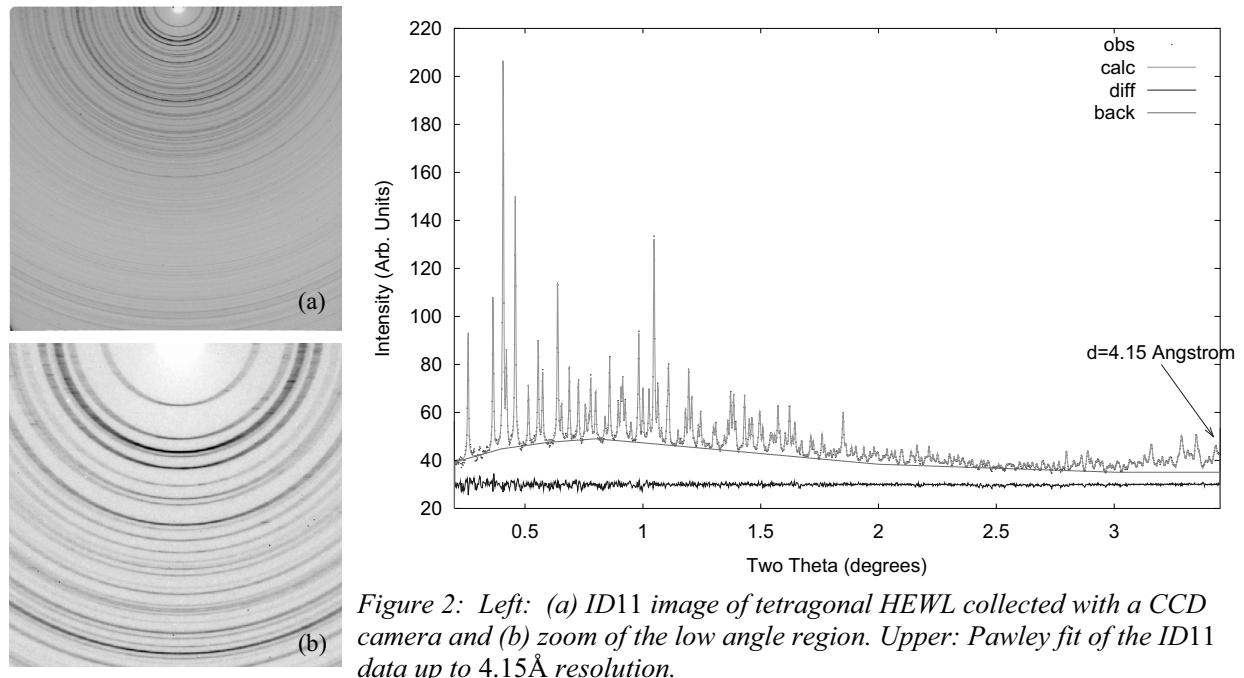

Figure 2: Left: (a) ID11 image of tetragonal HEWL collected with a CCD camera and (b) zoom of the low angle region. Upper: Pawley fit of the ID11 data up to $4.15 \AA$ resolution.

Area detectors allow for full powder rings to be recorded and integrated to yield gains of detection efficiency of more than $10^{3}$, leading to a drastic reduction in exposure times and radiation damage, thus making measurements on much smaller samples possible. The greatest advantage of using area detectors is that the solid angle for intensity integration increases 


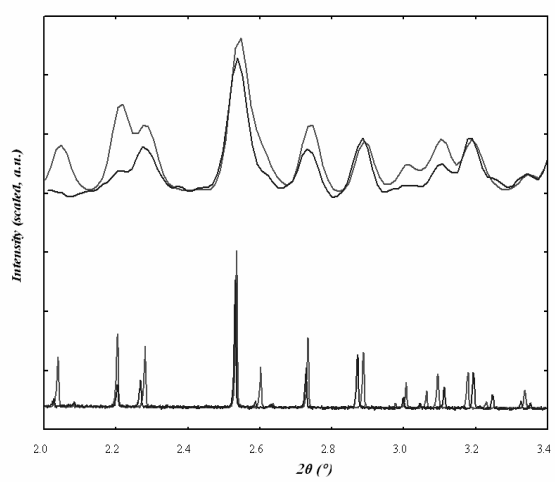

Figure 3: Low-angle region of powder diffraction patterns from lysozyme samples. The lower and upper patterns correspond to analyser crystal (ID31) and area detector (BM01A) data respectively. In these experiments, the intensities of certain low angle reflections are modulated by varying the mean electron density of the solvent surrounding the protein molecules in the crystal lattice via variations in the solvent composition. The red and blue spectra correspond respectively to samples soaked in low and high electron-dense solvents. with scattering angle resulting in improved intensity statistics in the high angle region of the powder data. This region suffers from significant decrease of the X-ray scattering amplitude (rate of intensity decrease $\propto \sin \theta / \lambda$ ) and requires longer data collection times for compensating the loss of information and achieving improved counting statistics. In collecting data with an area detector the main contributions to the peak widths come from the detector pixel size, the beam size at the detector and the sample dimensions. At any given instrument the detector pixel size is fixed and cannot be optimised for protein powder diffraction. The beam size at the detector can be minimised by arranging focused optics so that the beam is focused at the detector surface, as in a Guinier camera. Finally the contribution of the sample size increases with sample thickness and scattering angle $(\tan (2 \theta))$. In order to minimise the term due to sample size we have collected data at very high energies, $\sim 50 \mathrm{keV}$ $(0.25 \AA$ wavelength) at the materials sci-

ence beam line, ID11, at the ESRF. A $0.8 \mathrm{~mm}^{2}$ X-ray beam was vertically focused using a bent Laue crystal to give a beam size of $\sim 5 \mu^{2}$ at the surface of a Fast-Read-out-Low-Noise (FReLoN) $2 \mathrm{Kx} 2 \mathrm{~K}$ CCD camera [12], developed at ESRF, with $50 \mu \mathrm{m}^{2}$ pixel size. A $1 \mathrm{~mm}$ diameter sample of tetragonal HEWL was mounted at approximately $1.5 \mathrm{~m}$ in front of the detector. Figure 2 shows the CCD image and a zoom of the low angle region where the effects of X-ray beam focusing are particularly pronounced. A Pawley refinement, following integration of the data taking into account the middle $10^{\circ}$ and applying a median filter using the fit2d package [13], is also presented in figure 2 . The fitted peak width for these data is about $0.0042^{\circ}$ in $2 \theta$, equivalent to $\sim 0.021^{\circ}$ in $2 \theta$ at $1.25 \AA$, which is approximately two times larger with respect to the analyser crystal data. The peak shape function is Lorentzian in character and arises mainly from the point spread function of the detector. Due to the limited size of the area detector, the data only reach a minimum d-spacing of $4.15 \AA$. In order to improve these data, it will be necessary either to translate the area detector towards the sample at the expense of the FWHM-resolution or to employ a larger area detector.

In addition, measurements on a variety of model proteins have been performed at the SwissNorwegian beam line (BM01A). This station was initially designed for measurements on single crystals and features two different diffractometers with large-size area detectors. One is a multi-axis goniometer from Oxford Diffraction Ltd. equipped with a $165 \mathrm{~mm}$ diameter CCD detector. The second diffractometer consists of a single-axis goniometer combined with a $345 \mathrm{~mm}$ diameter image plate scanner from MAR-Research. The optical configuration of BM01A is a conventional arrangement of vertically collimating mirror, followed by a double crystal Si(111) monochromator and vertically focusing mirror. Sagittal bending of the second 


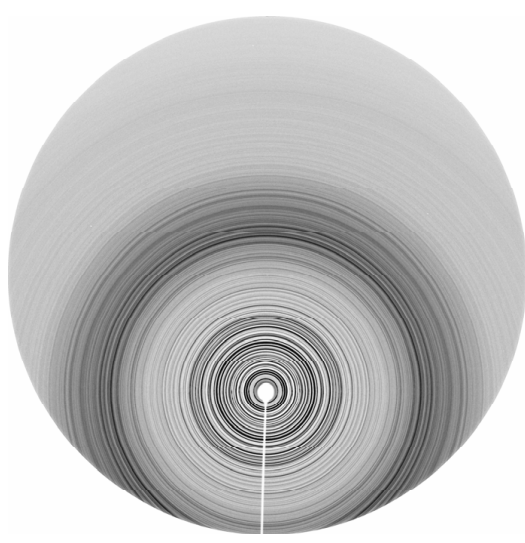

Figure 4: MAR345 image of HEWL crystallized from $1.25 \mathrm{M} \mathrm{NaCl} \mathrm{pH} 4.00 .05 \mathrm{M}$ phthalate buffer taken at $20 \mathrm{keV}$ as a $30 \mathrm{~s}$ exposure on beamline 1-BM at the $A d$ vanced Photon Source, Argonne National Laboratory; background from air, Kapton and solvent have been subtracted. Innermost ring is tetragonal HEWL (110) at $d$ $=55.66 \AA$, and the upper edge of the image corresponds to $d \sim 1.85 \AA$. monochromator crystal provides focusing in the horizontal plane. The angular resolution is typically a factor of 10 or worse in comparison with data collected on high-resolution instruments. However, many individual diffraction lines are still observable in protein powder diffraction patterns, especially in the low-angle region, and the indexing of such patterns is usually successful. Thus, areadetector data collection on protein powder samples is ideally suited for various qualitative and semi-quantitative measurements such as sample screening (i.e. checking for crystallinity), phase identification, time-resolved measurements, monitoring of phase transitions etc. As an example, in figure 3 , we present solvent contrast variation data recorded on powder samples of lysozyme, using both a highresolution diffractometer (ESRF-ID31) and an image-plate area detector (SNBL-BM01A). While the angular resolution from the area detector data is significantly worse, many lowangle diffraction lines are still identifiable and the effect of contrast variation is clearly visible in both pairs of powder patterns. It should be noted that the high-resolution powder patterns were recorded by irradiating a sample volume of approximately $3.5 \mathrm{~mm}^{3}$ for 15 minutes on an undulator beamline, whereas the area detector data were obtained by irradiating a sample volume of less than $0.5 \mathrm{~mm}^{3}$ for 6 minutes on a bending-magnet beamline.

Similarly, experiments at beam line 1-BM at the Advanced Photon Source (APS) take advantage of the full pattern recording capability of an image plate (MAR345) to collect rapidly protein powder diffraction data. In this case, the X-ray beam had energy of $20 \mathrm{keV}(\lambda \sim 0.62$ $\AA$ ) and was focused to the surface of the detector which was placed $\sim 700 \mathrm{~mm}$ from the sample position. [14]. The size of this focus spot $(\sim 300 \mu \mathrm{m})$ matches well with the MAR345 point spread function $(\sim 300 \mu \mathrm{m})$; the detector was then displaced vertically by $\sim 60 \mathrm{~mm}$ to enhance the accessible range of $\mathrm{d}$-spacings to $<2 \AA$. Figure 4 shows the image from the MAR345 detector of tetragonal HEWL.

In recent years, an increasing number of low-resolution protein structures has been determined by small angle scattering techniques [15]. The beam lines used for small angle scattering are usually similar to area detector beam lines discussed in this section but with a much longer sample to detector distance. During an in-situ crystallisation experiment we have recorded data for tetragonal HEWL at the small angle scattering beam line BM16 [16] at ESRF. Figure 5 shows an image from the MarCCD detector (pixel size $80 \mu \mathrm{m}^{2}$ ) which was placed $\sim 3 \mathrm{~m}$ behind the sample. The X-ray beam had a wavelength of $1 \AA$ and was focused at the surface of the detector using a toriodal mirror. After integrating the data using the fit $2 \mathrm{~d}$ package [13] the Pawley refinement shown in figure 5 indicates that the beam line was indeed suitable for powder diffraction. The fitted peak-widths are competitive with the high 

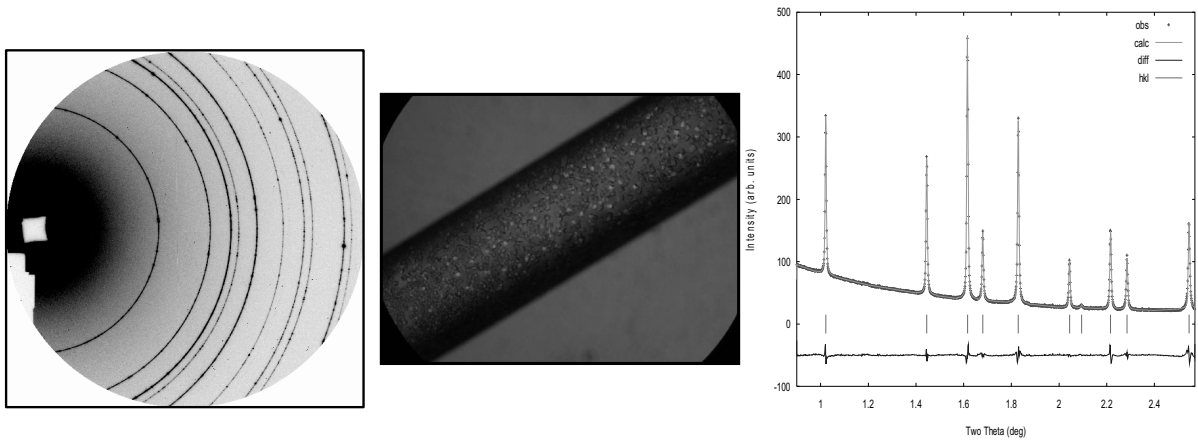

Figure 5: Left: Diffraction pattern of tetragonal HEWL recorded at the small angle scattering station, BM16. Middle: Picture taken on an optical microscope of tetragonal HEWL crystallised in a glass capillary ovenight during data collection at BM16. Right: Pawley fit to the integrated data (low angle region not shown). The final peak, at $2.545^{\circ}$ is the (221) reflection at d-spacing $22.5 \AA$.

resolution analyser crystal data, $\sim 0.004^{\circ}$, and the peak shape is fairly Lorentzian in shape with no detectable low-angle asymmetry contributions. The limited range in $2 \theta(22.5 \AA)$ means that only the first ten reflections could be recorded. These were, however, sufficient for phase identification and unit cell parameter refinement for the tetragonal unit cell.

The use of a long sample-to-detector distance in combination with a large Guinier-type camera has been particularly exploited at SPring8 with exciting results up to date [3b, 17]. The camera has a specimen-to-detector distance of $1000 \mathrm{~mm}$ with an evacuated path, and its angular resolution is $0.0029^{\circ} 2 \theta$ with an image plate (IP) reading pitch ( $50 \mu \mathrm{m}^{2}$ ). For reducing the point spread function of the detector, a Blue IP ( BAS-SR2040) is employed for high angular resolution data collection.
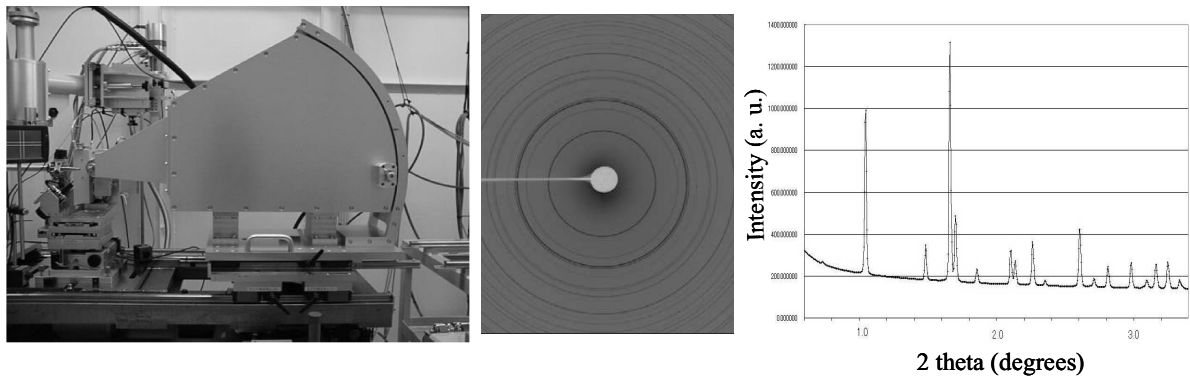

Figure 6: (left) the large Guinier-type camera at SPring8, (middle) zoom of the low angle region of the $2 D$ image of HEWL collected at BL40XU (SPring8) and (right) low-angle region of the integrated data.

The IP, with dimensions of $200 \mathrm{~mm}$ x $400 \mathrm{~mm}$, is read after exposure to the X-ray beam with an IP reader BAS2500 (Fuji film Co. (Ltd)) in an off-line manner. Initial studies were performed at the bending magnet beam line BL40B2 at SPring8, with a $(300 \mu \mathrm{m} \times 200 \mu \mathrm{m}) \mathrm{X}$ ray beam focused at the detector surface [3b]. In this case, measurements of tetragonal HEWL required 10 minutes exposure with a wavelength of $1 \AA$. Further work has been done 
at the high flux beam line BL40XU (SPring8), installed at an undulator source, which has a two focusing mirror system for achieving a highly parallel beam. A series of optical elements, including Si (220) channel-cut monochromator and a $25 \mu \mathrm{m}$ diameter pinhole with a $100 \mu \mathrm{m}$ diameter guard hole, have been added to further reduce the beam size $\left(50 \mu \mathrm{m}^{2}\right)$. Measurements on HEWL have been performed with a minimum instrumental contribution to the FWHM of around $0.003^{\circ} 2 \theta$ for $30 \mathrm{~s}$ exposure at $1 \AA$ wavelength. Figure 6 shows the image of tetragonal HEWL collected at BL40XU and the Guinier camera employed for data collection. Such long path length beam lines will be very well suited to the study of much larger proteins, where there are more low angle peaks to be found. This data collection indicates that the major limiting factor in the area detector beam lines is still the size of the detector itself. By moving the detector further back it is possible to obtain data which match analyser crystal FWHM-resolution and it should be possible to extend the range of the pattern by increasing the size of the detector.

\section{Laboratory instrumentation}

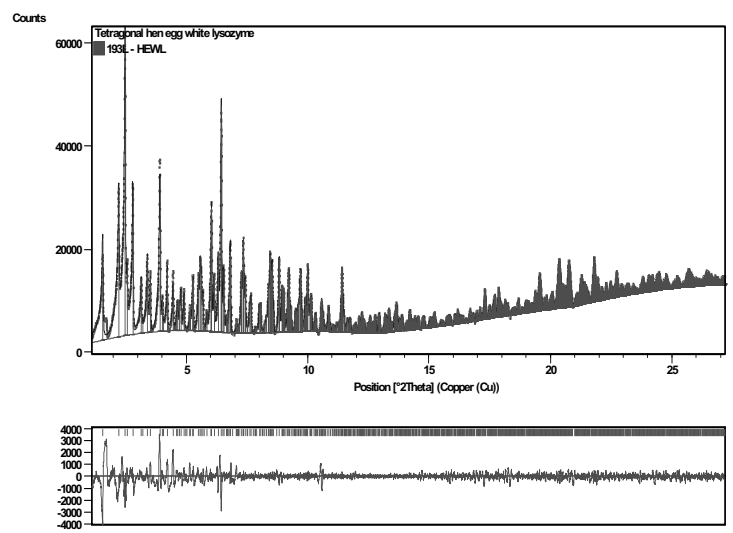

Figure 7: LeBail fit of data of tetragonal HEWL collected with an X'Pert PRO MPD laboratory X-ray powder diffractometer (PANalytical, The Netherlands).
Modern laboratory X-ray powder diffractometers offer the possibility to vary easily the instrumental resolution function and exchange beam geometries by adapting the $\mathrm{X}$ ray optics, analyzer crystals and detection systems. In this way it is possible to optimize quickly the instrumentation for a certain application. Even though previously it was impossible to measure small quantities of weakly scattering protein powder samples with high resolution optics in a reasonable time on a laboratory powder diffractometer, modern developments of new X-ray optical modules and detection systems allow for combining high intensity with high angular resolution. The data quality makes structural protein investigations such as indexing and unit cell refinements feasible and allows for the identification of polymorphic transformations and mixtures from data collected in a few hours. In addition, first attempts for structure refinements on protein powder laboratory data have been made with promising results. Although the lower intensity 

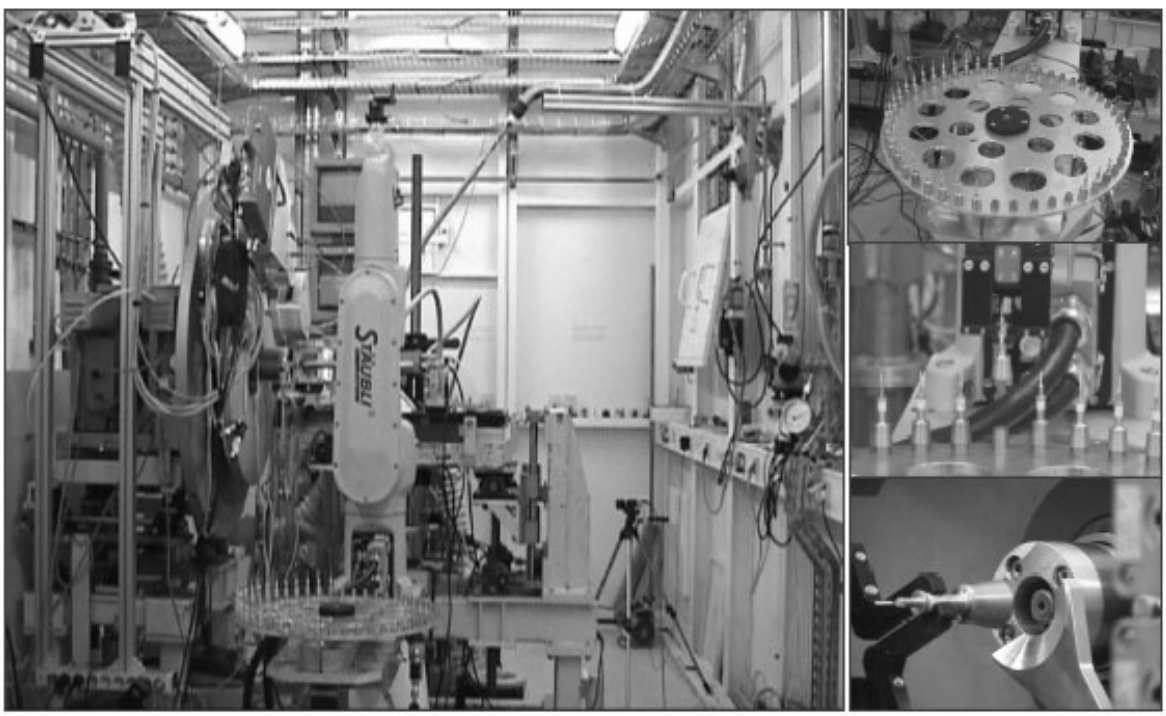

Figure 8: The automatic sample changing robot installed at ID31.

of a laboratory X-ray diffractometer requires significantly longer data collection times compared to a synchrotron, it has become an ideal tool for pre-screening of samples before they are analyzed in detail at one of the central X-ray facilities. In the case of the X'Pert PRO MPD laboratory X-ray powder diffractometer (PANalytical, The Netherlands), the X-ray multi-layer mirror focuses the incident beam $\left(\mathrm{Cu} \mathrm{K \alpha}\right.$-radiation) on the $\mathrm{X}^{\prime}$ Celerator detector, a linear solid-state silicon strip detector. This geometry allows combining a high-intensity beam with high angular resolution. Additional shielding reduces air scattering, especially important for weakly diffracting protein samples. In addition, 0.02 rad Soller slits are employed for reducing the axial divergence of the beam. Figure 7 shows the LeBail-fit of the data of a tetragonal HEWL sample collected with the instrumental configuration described above. The fitted peak width for these data is about $0.05^{\circ}$ in $2 \theta$. Indexing and LeBail fit were performed with the X'Pert HighScore Plus software [18].

\section{Crystal screening and principal component analysis (PCA)}

In recent years automated methods for crystal screening have been under development. One step towards this direction is the use of robotic equipment such as the automatic sample changer used for loading and unloading all the samples by mounting the capillaries in autocentring magnetic bases at ID31. Figure 8 shows the robotic equipment currently available. The efficiency of the apparatus was harnessed in the survey of the effect of $\mathrm{pH}$ on the crystallisation of HEWL, where 48 samples were measured overnight via the automated procedure [2b]. Figure 9 shows a colour representation of ID31 powder diffraction data from the $\mathrm{pH}$ variation experiment, from $\mathrm{pH} 6.56-3.33$ of $\mathrm{HEWL}$ crystallised at $4{ }^{\circ} \mathrm{C}$ and room temperature. At low temperature the tetragonal phase is favoured and a smooth anisotropic shift 

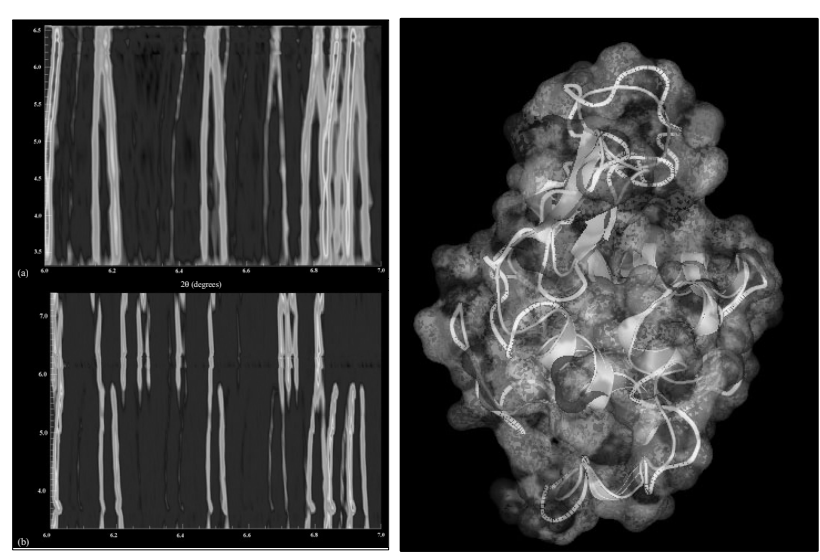

Figure 9: Left: Colour representation of ID31 powder diffration data from the $\mathrm{pH}$ variation experiment, from $\mathrm{pH} 6.56-3.33$ of HEWL crystallised at $4{ }^{\circ} \mathrm{C}$ (upper) and RT (lower). Right: Orthorcopic view of the refined molecular conformation of HEWL, shown as surface.

in the peak position is apparent whereas at room temperature the tetragonal (low $\mathrm{pH}$ ) to orthorhombic (high $\mathrm{pH}$ ) phase transition is observed at $\mathrm{pH} \sim 5.3$ [2b]. The refined molecular conformation of HEWL, as it was extracted from a 4-dataset stereochemically restrained Rietveld refinement performed using program GSAS [19], is shown as surface in figure 9.

Moreover, recent results obtained by directly exposing crystallisation trays to the X-ray beam suggest that powder diffraction can be considered as an alternative method to optical microscopy in crystal screening [20]. In this case, stainless steel plates containing 96 wells $(12 \times 8)$ were provided by PANalytical. These plates were prepared with tetragonal HEWL powder samples and positioned vertically in the X-ray beam on a translation stage at ID31. Mylar foils were used to reduce sample displacements. Each well contained approximately $2-5 \mu \mathrm{l}$ of protein precipitant and was exposed to a $1.0 \mathrm{~mm}^{2} \mathrm{X}$-ray beam with a wavelength of $1.250845(23) \AA$ for 2 minutes. Figure 10 shows the crystallisation tray and the data collected for one well filled with HEWL.

This kind of fast data collection requires a mutlivariate data analysis strategy for fingerprinting in order to compare similarities and differences between the various patterns corresponding to different samples. A successful method recently employed for the characterization of insulin microcrystals [4] is the principal component analysis (PCA). Several software packages $[18,21 \mathrm{a}-21 \mathrm{~b}]$ are already available that have incorporated, among other methods, PCA [21c] for the easy visualization of the total data variation of multiple powder diffraction patterns. The aim of this method is to arrange a series of diffraction patterns into clusters based on their mutual similarities allowing for dominant patterns and major trends to be rapidly obtained.

\section{Concluding remarks}

Each instrumental configuration discussed in the previous sections offers advantages in specific application domains. While it is tempting to try to obtain the highest resolution data (FWHM- angular resolution and resolution in d-spacing), a compromise is always forced upon us by the effects of radiation damage and the costs of producing large amounts of samples. In the process of screening extensive series of specimens under different conditions, the need to collect patterns rapidly from small samples is more important than obtaining data 
suitable for structure refinement. The unit cell parameters can normally be refined very pre-

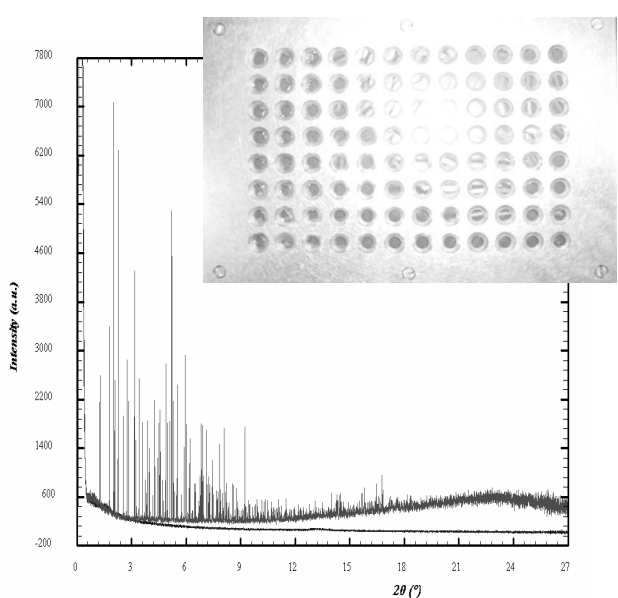

Figure 10: ID31 data $(\lambda=1.25 \AA, R T)$ of tetragonal HEWL (red line) and empty well (blue line). Inset: The crystallisation tray used for these measurements was provided by PANalytical. cisely from a relatively small $(<50)$ number of low angle peaks. The use of a lower-angular-resolution instrument allows constructing of phase diagrams and checking for ligand or heavy atom binding to be carried out rapidly using small samples. When particularly interesting samples are identified in the course of screening and a structure solution or refinement is to be attempted, it is of course critical to obtain the best possible data to increase the chances of success for the whole process. Here the analyser crystal beam lines offer unparalleled instrumental resolution which is very attractive in reducing the peak overlap. In comparison, multichannel position sensitive detectors, particularly those designed for larger unit cells, offer dramatically improved counting statistics. Ideally, both types of dataset should be available in a combined refinement in order to exploit the advantages of each. Special characteristics of protein powders require development of instrumentation to take real advantage of their inherent sharpness while at the same time avoid radiation damage effects. From one point of view, this could be achieved using high spatial resolution 2D area detectors designed to cover only the most useful part of the powder pattern (low $2 \theta$ region) and with increasing solid angle with $2 \theta$ to give the best possible statistics. We finally observe that the most significant improvements have recently come from the use of multiple datasets during intensity extraction [9] and/or structure refinements [1f, 2b, 22]. The unit cell parameters are perturbed to alter the pattern of peak overlaps in the different patterns and reduce the overlap problem. Ultimately, in the cases that this approach can be pursued it is likely to lead to enhanced results in comparison to a single pattern analysis.

\section{References}

1. (a) Von Dreele, R.B., 1999, J. Appl. Cryst., 32, 1084-1089. (b) Von Dreele, R.B. et al. 2000, Acta Cryst., D56, 1549-1553. (c) Von Dreele, R.B., 2001, Acta Cryst., D57, 1836-1842. (d) Von Dreele, R.B., 2003, Method. Enzymol., 368, 254-267. (e) Von Dreele, R.B., 2005, Acta Cryst., D61, 22-32. (f) Von Dreele, R. B., J. Appl. Cryst. (submitted).

2. (a) Margiolaki, I., et al. 2005, Acta Cryst., D61, 423-432. (b) Basso, S. et al. 2005, Acta Cryst., D61, 1612-1625. 
3. (a) Balbirnie, M. et al. 2001, PNAS, 98, 2375-2380. (b) Diaz Avalos, R. et al. 2003, J. Mol. Biol., 330, 1165-1175. (c) Bond J. P. et al. 2003, J. Str. Biology, 141, 156170. (d) Anduleit, K. et al. 2005, Protein Sci., 14, 2741-2743.

4. Norrman M. et al. 2006, J. Appl. Cryst., 39, 391-400.

5. Jenner, M. et al. J. Appl. Cryst. (In Press).

6. Fitch, A. N., 2004, J. Res. Natl. Inst. Stand. Technol., 109, pp. 133-142.

7. (a) Patterson, B. D. et al. 2005, Nuclear Instr. Methods, A540, 42-67. (b) Schmitt B. et al. 2004, Nucl. Instr. and Meth., A518, 436-439. (c) Gozzo, F. et al. 2004, Journal of Alloys and Compounds, 362, 206-217. (d) Gozzo, F. et al. 2006, J. Appl. Cryst., 39, 347-357.

8. (a) Murray, J. W. et al. 2004, J. Appl. Cryst., 37, 513-522. (b) Murray, J. W. et al. 2005, J. Synch. Rad., 12, 268-275 (2005). (c) Nave, C. et al. 2005, J. Synch. Rad., 12, 257-260.

9. Besnard, C. et al. Z. Kristallogr. (Submitted).

10. Pawley, G.S., 1981, J. Appl. Cryst., 14, 357-361.

11. Bruker AXS (2003): TOPAS V2.1: General profile and structure analysis software for powder diffraction data. - User's Manual, Bruker AXS, Karlsruhe, Germany.

12. Labiche, J. C. et al. 1996, FReLoN camera: Fast readout and low noise, ESRF newsletter no 25, 41-43.

13. Hammersley, A. P. et al. 1996, High Pressure Research, 14, 235-248.

14. Von Dreele, R.B. et al. 2006, Z. Kristallogr. Suppl., 23, 3-8.

15. Svergun, D. I., Hoch, M. J., 2002, Current Opinion in Structural Biology, 12, 654660.

16. Rueda, D. R. et al. 2006, Rev. Sci. Instrum., 77, 033904-033908.

17. Miura, K. et al. 2004, AIP Conference Proceedings 705, 989-992.

18. PANalytical: X'Pert Highscore Plus V2.2a (http://www.panalytical.com/index.cfm?pid=547).

19. Larson, A. C., Von Dreele, R. B., 2004, General Structure Analysis System (GSAS), Los Alamos National Laboratory Report LAUR, 86-748.

20. Jacquamet, L. et al. 2004, Structure, 12, 1219-1225.

21. (a) Wold, S. et al. 1987, Chemom. Intell. Lab. Syst., 2, 37-52. (b) Barr, G. et al. 2004, J. Appl. Cryst., 37, 658-664. (c) Joliffe, I. T., 1986, Principal Component Analysis, Springer-Verlag, New York.

22. (a) Zachariasen, W. H. et al. 1963, Acta Cryst., 16, 369-375. (b) Shankland, K. et al. 1997, J. Mater. Chem., 7, 569-572. (c) Brunelli, M. et al. 2003, Angew. Chem. Int. Ed., 42, (18), 2029-2032.

Acknowledgements. We are grateful to ESRF, APS, SPring8 and SLS for provision of beamtime. Use of the APS was supported by the US DOE/OS/OBES, Contract No.W-31109-ENG-38. We thank PANalytical for extensive use of lab diffractometers and the provision of software for PCA analysis. We also thank Peter Stephens for useful discussions. 